\title{
Do-it-yourself radiative cooler as a radiative cooling standard and cooling component for device design
}

\author{
Xin Huang, Jyotirmoy Mandal $\odot$,* and Aaswath P. Raman* \\ University of California, Los Angeles, United States
}

\begin{abstract}
We demonstrate a simple, low-cost design of a selectively emissive radiative cooler using scotch tape and aluminum foil, which can be further augmented by higher quality metal deposition methods. This do-it-yourself radiative cooler achieves solar reflectance, long wavelength infrared emittance, and optical selectivity comparable to state-of-the art designs and is experimentally demonstrated as achieving a $7^{\circ} \mathrm{C}$ subambient temperature drop at night for the aluminized scotch tape and an average $2^{\circ} \mathrm{C}$ drop under a solar illumination of $965 \mathrm{~W} / \mathrm{m}^{2}$ for the silvered scotch tape. In addition, an $11^{\circ} \mathrm{C}$ subambient temperature drop at night for the aluminized scotch tape was obtained when a convection shield was used. Detailed optical properties are presented for an ultrawide wavelength range and a $\sim 2 \pi$ angle of emittance. Given its ease of fabrication and performance, we propose this set of materials as a control for future radiative cooling experiments and an effective radiative cooling accessory for passive cooling designs. () 2021 Society of Photo-Optical Instrumentation Engineers (SPIE) [DOI: 10.1117/1.JPE.12.012112]
\end{abstract}

Keywords: do-it-yourself; low-cost; radiative cooling; scotch tape; selective thermal emitter; standard reference.

Paper 21063SS received Aug. 7, 2021; accepted for publication Oct. 26, 2021; published online Nov. 17, 2021.

\section{Introduction}

Passive radiative cooling, which involves net heat loss from terrestrial objects to the cold of outer space through long wavelength infrared (LWIR, $\lambda \sim 8$ to $13 \mu \mathrm{m}$ ) transmission windows of the atmosphere, is a zero-energy, zero-carbon method of cooling objects under the sky. In recent years, it has been increasingly explored ${ }^{1-12}$ as an alternative or complement to active cooling methods for a range of applications, including cooling buildings, ${ }^{13}$ harvesting dew, ${ }^{14-16}$ and thermoelectric power generation. ${ }^{17} \mathrm{~A}$ variety of materials and designs, ranging from photonic architectures ${ }^{18}$ to scalable polymer films, ${ }^{19-21}$ paints, ${ }^{22,23}$ and composites, ${ }^{24}$ have been created for radiative cooling. These materials and designs are capable of efficient radiative cooling and are typically suited to specific applications. However, the simplicity of design and ease of application remain major research endeavors-both for scientific and large-scale uses. Low-cost radiative coolers ${ }^{25}$ have attracted much attention recently with the possibility of greatly expanding the scope of radiative cooling's deployment.

In this article, we demonstrate an easily fabricable and efficient radiative cooling design made using household materials. The radiative cooler can be made from scotch tape and aluminum foil and has excellent infrared optical characteristics for radiative cooling: selective LWIR emittance [ $\varepsilon_{\text {LWIR }}$, emittance in the 8 to $13 \mu \mathrm{m}$ (LWIR) wavelength range] of 0.87 at near-normal incidence and hemispherical selective LWIR emittance of 0.83 . Furthermore, it is flexible, robust, scalable, and low-cost. The total broadband emittance $\varepsilon_{\mathrm{BB}}$ of scotch tape is 0.68 at near-normal incidence, and its hemispherical broadband emittance is 0.63 . The back aluminum foil yields a modest weighted solar reflectance at 0.83 . If silver is used as a back reflector, the design's high solar reflectance $\left(R_{\text {solar }} \sim 0.95\right)$ makes it capable of daytime radiative cooling as well. We experimentally demonstrate a subambient cooling by $11^{\circ} \mathrm{C}$ at night with a convection shield and $7^{\circ} \mathrm{C}$ without a convection shield and daytime cooling performance with a $2^{\circ} \mathrm{C}$ drop under a solar illumination of $965 \mathrm{Wm}^{-2}$ without any convection shield. Given that the design has high optical performance and is created using standard commercially available materials, we argue that this

*Address all correspondence to Jyotirmoy Mandal, jyotirmoymandal@ucla.edu; Aaswath P. Raman, aaswath@ucla.edu 
makes it attractive as a convenient and reproducible standard-both for designs that require a generic radiative cooling component for their operation and as a control for radiative cooling experiments. We highlight its high LWIR emittance, along with the modest directional LWIR selectivity at near-normal incidence, defined as $\frac{\varepsilon_{\mathrm{LWIR}}(\text { near-normal incidence) }}{\varepsilon_{\mathrm{BB}} \text { (near-normal incidence) }}$ of 1.28 , and hemispherical LWIR selectivity, defined as $\frac{\varepsilon_{\mathrm{LWIR}} \text { (hemispherical) }}{\varepsilon_{\mathrm{BB}} \text { (hemispherical) }}$ of 1.32 . The intermediate nature of its selectivity may also make it useful as a reference threshold between broadband and selective radiative coolers. Toward these ends, we provide extensive optical characterization for the radiative cooling research community to use as a reference.

\section{Results}

We investigated scotch-tape-based radiative cooling designs because of their readily reproducible optical performance, which arises from their standardized material constituents. To achieve passive radiative cooling, a radiative cooling design should have a high emittance $\varepsilon$ in the LWIR atmospheric transmission window $(\lambda \sim 8$ to $13 \mu \mathrm{m})$ and a high $R_{\text {solar }}$ for subambient cooling during the day. In this regard, certain variants of scotch tapes, which comprise acrylic adhesive on a polypropylene film, are intuitive choices [Fig. 1(a)]. Both acrylic and polypropene have chemical bonds that absorb and hence radiate heat in the LWIR wavelengths, which can be lost to space [Fig. 1(b)]. A lack of highly emissive chemical bonds in the non-LWIR thermal wavelengths makes sufficiently thin films of both materials selectively LWIR emissive. The 3M Long Lasting Scotch Tape, which we propose for use here, combines both these properties in a standardized and reproducible manner.

(a)

(b)
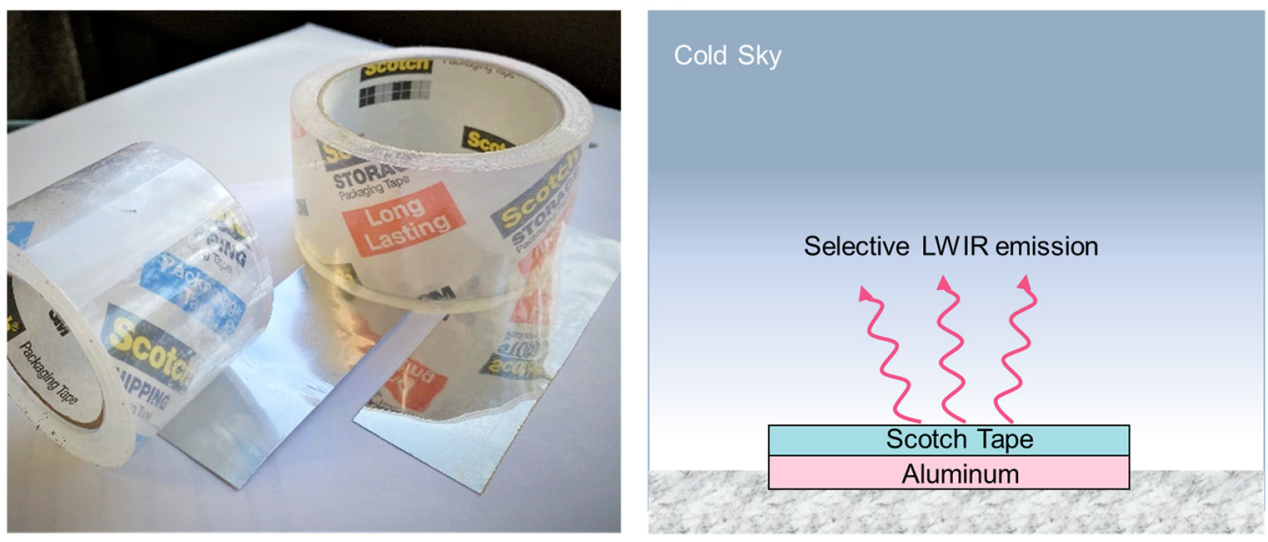

(c)

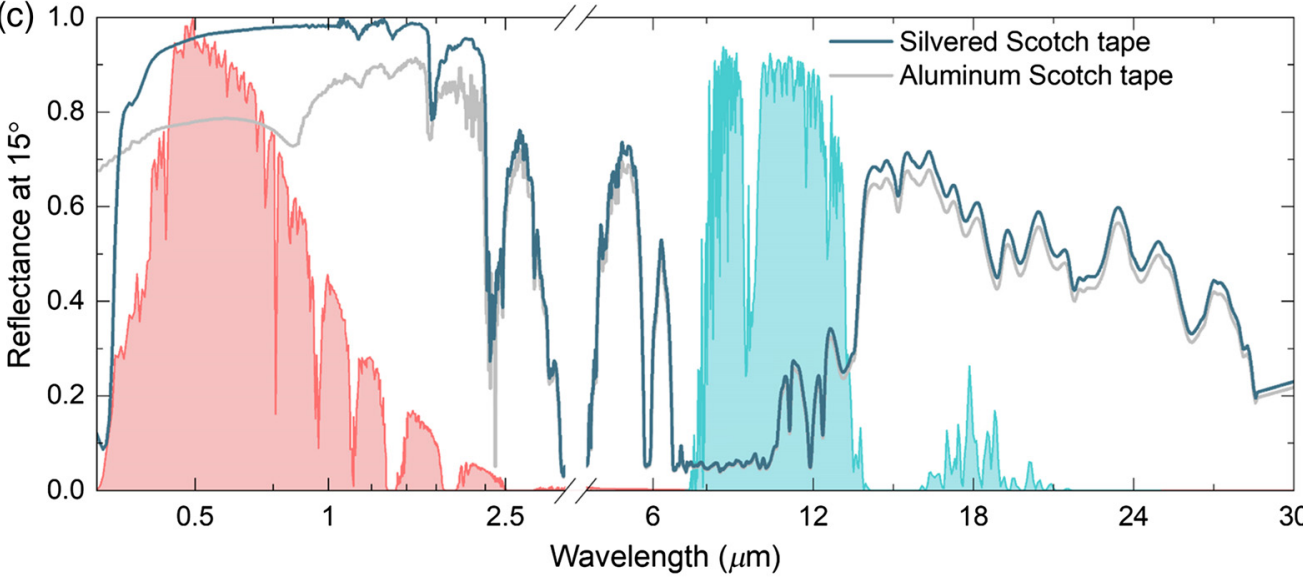

Fig. 1 (a) Photo of silvered scotch tape. Two-layer scotch tape is coated with silver on the bottom. (b) Schematic of the scotch tape radiative cooler with aluminum. (c) Reflectance of silvered scotch tape and aluminized scotch tape at 15-deg incident angle. 
As an infrared reflector for the scotch tape, we use commercially available Reynolds Wrap aluminum foil, although we note that aluminum foils from other brands have similar infrared reflectance. A design comprising two layers of tape on aluminum foil has spectral reflectance shown in Fig. 1(c) for near-normal incidence. The ultrawide bandwidth spectral measurements were taken using a Perkin Elmar Lambda 950 spectrophotometer and a directional selectivity of 1.28 and a modest $R_{\text {solar }}$ of 0.83 . If sputter-coated silver is used instead of aluminum, the solar reflectance rises to 0.95 [Fig. 1(c)].

The optical performance that we highlight here, even without considering the simplicity of the design, is noteworthy in two ways. First, the scotch-tape-based design combines a moderately selective infrared emittance with a reasonably high, near-normal $\varepsilon_{\text {LWIR }}$. This makes it useful as a nighttime radiative cooler and, while not a subambient radiative cooling design under strong sunlight, a point of reference nonetheless for experiments involving other radiative coolers. Second, when backed with sputter-coated silver, its solar reflectance (0.95) is sufficiently high for daytime radiative cooling under most meteorological conditions [Fig. 1(c)]. The optical parameters of this radiative cooler design are thus quite good and rank highly among known designs.

A more detailed picture of the scotch tape's radiative capabilities is revealed by the hemispherical emittance [Fig. 2(a)] derived from angular measurements [Fig. 2(b)] of reflectance at 15 deg to 84 deg using a Harrick reflectance measurement accessory coupled with an FTIR (Bruker Invenio-R) and averaged using Eq. (1):

$$
\varepsilon_{\lambda}=\frac{\int_{0}^{2 \pi} \int_{0}^{\pi / 2} \varepsilon_{\lambda, \theta}(\lambda, \theta, \varphi) \cos \theta \sin \theta \mathrm{d} \theta \mathrm{d} \varphi}{\int_{0}^{2 \pi} \int_{0}^{\pi / 2} \cos \theta \sin \theta \mathrm{d} \theta \mathrm{d} \varphi} .
$$
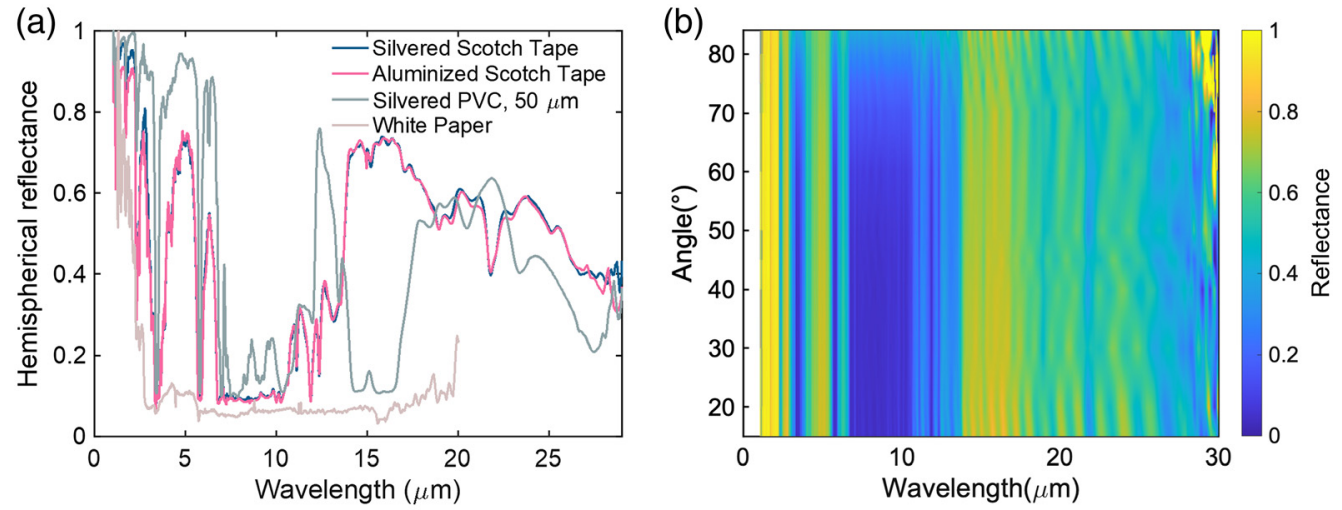

(c)

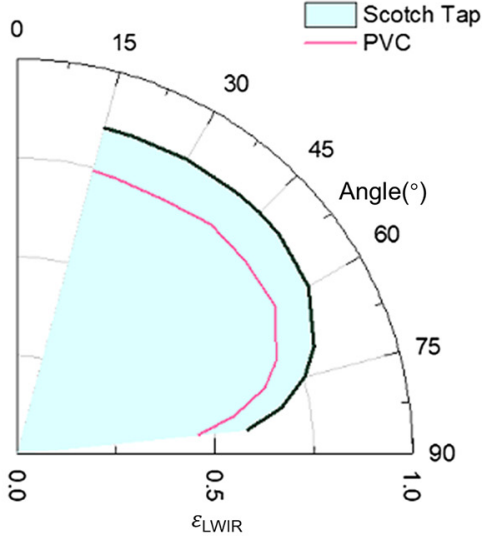

(d)

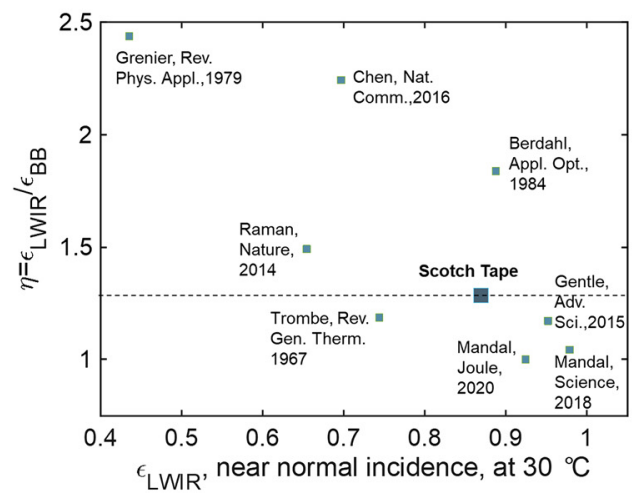

Fig. 2 (a) Spectral reflectance $\lambda=1-\varepsilon(\lambda)$ of the silvered scotch-tape radiative cooler, presented alongside that of metallized PVC proposed by Trombe (reproduced by silvering a $50-\mu \mathrm{m}$ film) and white paper. (b) Emittance of the silvered scotch-tape radiative cooler from 15 deg to 84 deg. (c) Angular emittance ( $=1$ - reflectance) of the two designs in Fig. 2(a). (d) $\varepsilon_{\mathrm{LWIR}}$ and $\boldsymbol{\eta}$ of silvered scotch-tape radiative cooler compared against notable designs in the literature. ${ }^{3,18-22,26,27}$ 
We observe that scotch tape has a hemispherical emittance in the LWIR atmospheric window that is high (0.83) and selective. It is difficult for us to compare the performance directly with similar polymer films in the literature as data are scarce. However, we note that it compares favorably with the hemispherical emittance of a highly scalable polymer based radiative cooler ( $\sim 0.80)$ proposed by Angus and Gentle ${ }^{19}$ and that of our reproduction of metallized polyvinyl chloride (0.73), which was the first polymeric radiative cooler demonstrated by Trombe ${ }^{21}$ [Fig. 2(a)]. Hemispherical reflectance of white paper, a common broadband thermal emitter, is also shown in Fig. 2(a), comparison with which highlights the LWIR selective emittance of the scotch tape. The $\varepsilon_{\text {LWIR }}$ of scotch tape persists at high angles, as shown in Fig. 2(c), which enables it to lose heat through the spatial extent of the LWIR window, which only closes near the horizontal.

We also investigate the scotch tape's performance relative to notable broadband emitter and selective emitters in the literature [Fig. 2(d)]. The $\varepsilon_{\text {LWIR }}$ of scotch tape at near-normal incidence is 0.87 , which is fairly high, while its directional selectivity at near-normal incidence is 1.28 , which appears to fall between highly selective ${ }^{18,21}$ and broadband designs. ${ }^{3,19,22}$ It should be noted here that the performances compared are for near-normal emittances. Further data are provided in an online archive. ${ }^{28}$

The optical parameters, which are suitable for radiative cooling, lead to good radiative cooling performance during both day and night. Daytime performance of scotch tape was demonstrated by exposing it directly under the sun in Los Angeles, California, on March 27, 2021, as is shown in Fig. 3(a). The scotch tape can maintain a $2^{\circ} \mathrm{C}$ to $3^{\circ} \mathrm{C}$ temperature drop compared with the ambient air temperature under $965 \mathrm{~W} / \mathrm{m}^{2}$ solar radiation. We also demonstrated the

(a)

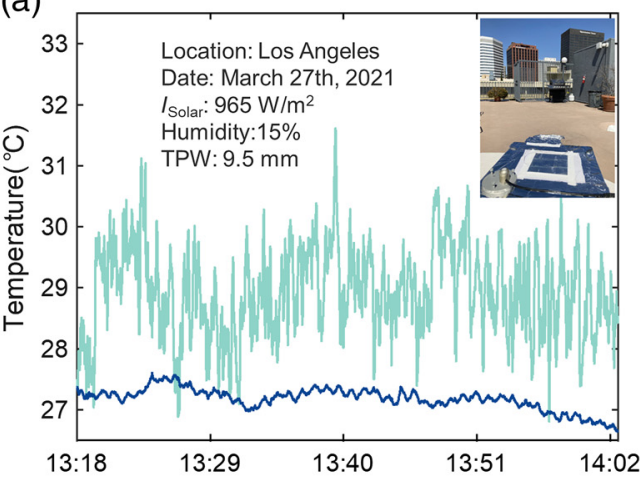

(b)

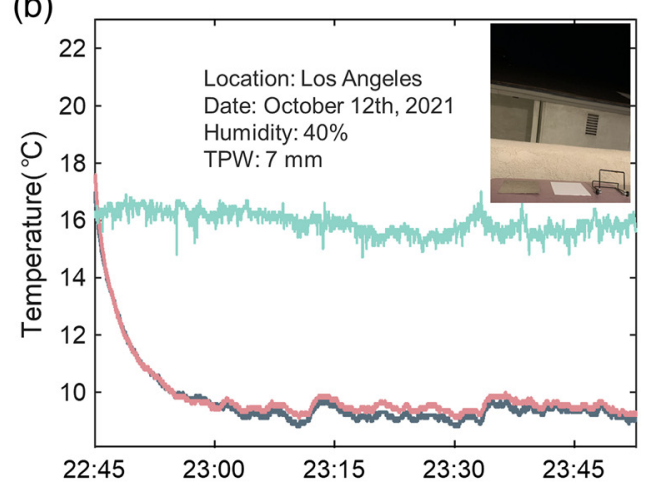

(c)

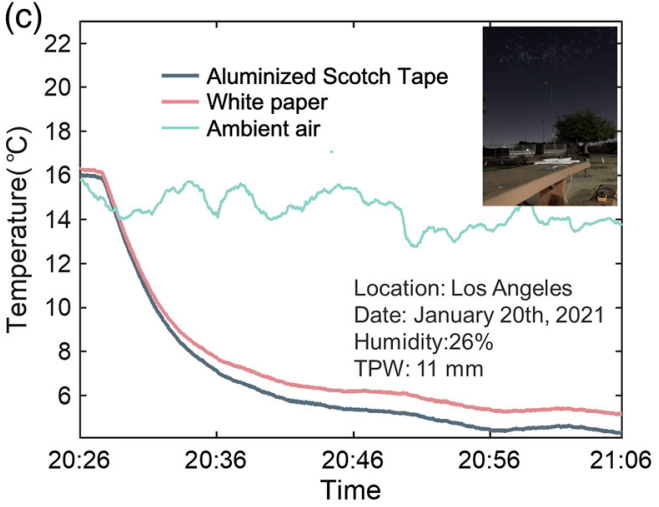

Fig. 3 (a) Measurement of the silvered scotch tape's temperature (blue) against ambient air temperature (green) on a clear day in Los Angeles, California, on March 27, 2021. (b) Measurement of the aluminized scotch tape's temperature (blue) and double-layer white paper's temperature (pink) against ambient air temperature (green) on a clear night in Los Angeles, California, on October 12, 2021. (c) Measurement of the aluminized scotch tape's temperature (blue) and double-layer white paper's temperature (pink) against ambient air temperature (green) on a clear night with convection shield in Los Angeles, California, on January 20, 2021. 
nighttime performance of the scotch tape in Los Angeles, California, on October 12, 2021, by exposing it to the sky during nighttime hours and testing its performance compared with the broadband thermal emitter, white paper. As shown in the temperature data of Fig. 3(b), immediately after the scotch tape is exposed to the environment [22:45 local time in Fig. 3(b)], its temperature drops $\sim 7^{\circ} \mathrm{C}$ below the measured ambient air temperature. Compared with the white paper, which is a broadband emitter with an emittance of $\sim 0.9$ [Fig. 2(a)], the scotch tape is $\sim 0.3^{\circ} \mathrm{C}$ cooler despite its lower hemispherical emittance. To highlight the effect of selectivity, another nighttime experiment was conducted with a single layer low density polyethylene convection shield in Los Angeles, California, on January 20, 2021, by exposing it to the sky during nighttime hours. As is shown in Fig. 3(c), compared with the white paper, the scotch tape is $1^{\circ} \mathrm{C}$ cooler. It should be noted that both the higher nighttime cooling performances by the scotch tape are attributable to its selective emittance as the broadband paper emitter has a higher LWIR emittance than scotch tape.

\section{Discussion}

To summarize, we have characterized and demonstrated the optical performance of a scotchtape-based radiative cooler, which can be deployed at scale for passive cooling applications. With the scotch tape and aluminum foil, it can have a $7^{\circ} \mathrm{C}$ temperature drop compared with ambient temperature during night, which makes it useful for cooling down the objects and generating electricity using this temperature difference. A large temperature drop of $11^{\circ} \mathrm{C}$ is achieved using a low-density polyethylene convection shield. The silvered scotch tape can be used for daytime cooling with a $2^{\circ} \mathrm{C}$ to $3^{\circ} \mathrm{C}$ temperature drop, which could make it a good candidate for applications requiring modest subambient cooling.

Unlike other approaches to making radiative coolers, which require new or expensive materials and fabrication techniques, scotch tape can be directly applied on the aluminum foil at low cost. Furthermore, the mass production and wide availability of scotch tape make it a reliable standard as a thermal emitter, and coupling it with commercial aluminum foil or vapor deposited silver makes it a reproducible radiative cooler that could be used to standardize outdoor radiative cooling experiments. This stands in contrast to other commonly used radiative cooling polymers such as PDMS, ${ }^{4,29}$ which are efficient radiative coolers but are hard to coat or otherwise apply in a reproducible manner. Therefore, we propose the following.

1. It can be used as a standard reference radiative cooler-with the aluminized version being sufficient as a generic radiative cooling standard and the silvered version being a standard for subambient daytime applications. We would also like to note that, although aluminized scotch tape cannot achieve subambient cooling under strong sunlight, because it has been well-characterized, it can still be a useful point of reference to verify other measurements and environmental variables for daytime experiments. The low cost and easy fabrication of such a standard could allow it to easily be used by researchers and practitioners as a control in outdoor experiments. It should be noted that while the aluminized version can be made using standard commercial materials and is thus highly reproducible, the silvered variant's solar reflectance may depend on the silver deposition process and requires a high quality, optically thick $(>200 \mathrm{~nm})$ coating.

2. Because there currently exist no clear boundaries delineating selective and broadband thermal emitters, we propose that the scotch tape radiative cooler could serve as a threshold of selectivity. Materials with selective emittance ratios higher than that of scotch tape can be considered as the selective thermal emitters and others as broadband thermal emitters.

3. It can be used as an easily fabricable radiative cooler for investigations in which a radiative cooling material itself is not central to the study. Potential examples of this include thermoelectricity generation, dew collection, and desalination using radiative cooling, in which metallized scotch tape can be used as a cooling component.

Toward these ends, we have shown the full spectrum ranging from 0.3 to $30 \mu \mathrm{m}$ for silvered and aluminized scotch tapes and angular emittances for $15 \mathrm{deg}$ to $84 \mathrm{deg}$ for use as a reference. 
The data, which, to our knowledge, represent the most extensive characterization of a radiative cooler, are publicly available online. ${ }^{30,31}$ The data can also be used for theoretical simulations of radiative coolers. With radiative cooling emerging as an important frontier for research, we hope that this work can offer a potential standard control for other radiative coolers and radiative cooling experiments.

\section{Acknowledgments}

We acknowledge support of the UCLA Hellman Fellows Award. J. M. was supported by the Schmidt Science Fellows program, in partnership with the Rhodes Trust.

\section{References}

1. L. Zhu, A. Raman, and S. Fan, "Color-preserving daytime radiative cooling," Appl. Phys. Lett. 103, 223902 (2013).

2. L. Zhu, A. P. Raman, and S. Fan, "Radiative cooling of solar absorbers using a visibly transparent photonic crystal thermal blackbody," Proc. Natl. Acad. Sci. U. S. A. 112, 12282-12287 (2015).

3. Y. Zhai et al., "Scalable-manufactured randomized glass-polymer hybrid metamaterial for daytime radiative cooling," Science 355, 1062-1066 (2017).

4. J. Kou et al., "Daytime radiative cooling using near-black infrared emitters," ACS Photonics 4, 626-630 (2017).

5. E. A. Goldstein, A. P. Raman, and S. Fan, "Sub-ambient non-evaporative fluid cooling with the sky," Nat. Energy 2, 17143 (2017).

6. B. Bhatia et al., "Passive directional sub-ambient daytime radiative cooling," Nat. Commun. 9, 5001 (2018).

7. H. Kim and A. Lenert, "Optical and thermal filtering nanoporous materials for sub-ambient radiative cooling," J. Opt. 20084002 (2018).

8. D. Zhao et al., "Subambient cooling of water: toward real-world applications of daytime radiative cooling," Joule 3, 111-123 (2019).

9. P. Yang, C. Chen, and Z. M. Zhang, "A dual-layer structure with record-high solar reflectance for daytime radiative cooling," Sol. Energy 169, 316-324 (2018).

10. B. Zhao et al., "Radiative cooling: a review of fundamentals, materials, applications, and prospects," Appl. Energy 236, 489-513 (2019).

11. A. W. Harrison and M. R. Walton, "Radiative cooling of $\mathrm{TiO}_{2}$ white paint," Sol. Energy 20, 185-188 (1978).

12. A. Hjortsberg and C. G. Granqvist, "Radiative cooling with selectively emitting ethylene gas,” Appl. Phys. Lett. 39, 507-509 (1981).

13. J. Mandal et al., "Radiative cooling and thermoregulation in the Earth's glow," arXiv:2006. 11931 (2021).

14. W. Li et al., "Nighttime radiative cooling for water harvesting from solar panels," $A C S$ Photonics 8, 269-275 (2021).

15. M. Zhou et al., "Vapor condensation with daytime radiative cooling," Proc. Natl. Acad. Sci. U. S. A. 118, e2019292118 (2021).

16. I. Haechler et al., "Exploiting radiative cooling for uninterrupted 24-hour water harvesting from the atmosphere," Sci. Adv. 7, eabf3978 (2021).

17. A. P. Raman, W. Li, and S. Fan, "Generating light from darkness," Joule 3, 2679-2686 (2019).

18. A. P. Raman et al., "Passive radiative cooling below ambient air temperature under direct sunlight," Nature 515, 540-544 (2014).

19. A. R. Gentle and G. B. Smith, "A subambient open roof surface under the mid-summer sun," Adv. Sci. 2, 1500119 (2015).

20. P. Grenier, "Réfrigération radiative, effet de serre inverse," Rev. Phys. Appl. 14, 87-90 (1979).

21. F. Trombe, "Perspectives sur l'utilisation des rayonnements solaires et terrestres dans certaines régions du monde," Rev. gén. Therm. 6, 1215-1234 (1967). 
22. J. Mandal et al., "Hierarchically porous polymer coatings for highly efficient passive daytime radiative cooling," Science 362, 315-319 (2018).

23. J. Mandal et al., "Paints as a scalable and effective radiative cooling technology for buildings," Joule 4, 1350-1356 (2020).

24. A. R. Gentle and G. B. Smith, "Radiative heat pumping from the earth using surface phonon resonant nanoparticles," Nano Lett. 10, 373-379 (2010).

25. L. Zhou et al., "A polydimethylsiloxane-coated metal structure for all-day radiative cooling," Nat. Sustain. 2, 718-724 (2019).

26. Z. Chen et al., "Radiative cooling to deep sub-freezing temperatures through a 24-h day-night cycle," Nat. Commun. 7, 13729 (2016).

27. P. Berdahl, "Radiative cooling with $\mathrm{MgO}$ and/or LiF layers," Appl. Opt. 23, 370-372 (1984).

28. J. Mandal, "Survey of radiative coolers," Tableau Public, 2021, https://public.tableau.com/ app/profile/jyoti.mandal/viz/SurveyofRadiativeCoolers-TestVersion/Homepage (accessed November 2021).

29. B. Czapla et al., "Potential for passive radiative cooling by PDMS selective emitters," in Heat Transfer Summer Conf., American Society of Mechanical Engineers Digital Collection (2017).

30. J. Mandal, "Resources for radiative cooling research," Jyotirmoy Mandal, 2021, https:// jyotirmoymandal.com/radiative-cooling-resources/ (accessed November 2021).

31. A. P. Raman, "Raman Lab @ UCLA,” https://github.com/Raman-Lab-UCLA

Xin Huang is a PhD student in the Materials Science and Engineering Department, University of California, Los Angeles. He joined Prof. Raman's lab in 2019 to work on controlling thermal emission, focusing on fundamental mechanisms and applications to water and energy technologies.

Jyotirmoy Mandal completed his $\mathrm{PhD}$ in applied physics at Columbia University in New York City, and is currently a Schmidt science fellow and a postdoctoral researcher at University of California, Los Angeles. His research interests include low-cost optical designs for radiative cooling and solar heating, with a focus on applications in developing countries.

Aaswath P. Raman is an assistant professor of materials science and engineering at the University of California, Los Angeles (UCLA). He received his $\mathrm{PhD}$ in applied physics from Stanford University in 2013. His research interests span nanophotonics, computational electromagnetics, solid-state devices, and energy applications, including radiative cooling, where he is known for pioneering demonstrations of daytime radiative cooling. His group at UCLA explores these and related topics from a multidisciplinary perspective. 